\title{
On the sums of continued fractions
}

by

Bomustav Drviš (Columbus, Ohio)

Dedicated to the memory of my teacher Professor Vojtěch Jarnik

Let $\alpha$ be an irrational number and $\alpha=\left(g_{0} ; g_{1}, g_{2}, \ldots\right)$ its regular continued fraction expansion. For each natural number $N$ we shall denote by $F_{N}$ the set of all irrational numbers $\alpha$, for which $g_{j} \leqslant N \quad(j=1,2,3$, ..). M. Hall Jr. [1] has proved that each real number $\beta$ can be written in the form $\beta=\alpha_{1}+\alpha_{2}$, where $\alpha_{j} \in F_{4}(j=1,2)$. The purpose of this paper is to show that each real number can be expressed as the sum of three elements of the set $F_{3}$ or four elements of the set $F_{2}$. Moreover, we show that a lesser number of summands, in general, does not suffice.

THEORem 1. Let $\beta$ be a real number. Them there exist three numbers $\alpha_{j} \in F_{3}(j=1,2,3)$ such that $\beta=\alpha_{1}+\alpha_{2}+\alpha_{3}$. Also there exist real numbers $\beta^{\prime}$ such that $\beta^{\prime} \neq \alpha_{1}^{\prime}+\alpha_{2}^{\prime}$ for any pain $\left(\alpha_{1}^{\prime}, \alpha_{2}^{\prime}\right), \alpha_{i}^{\prime} \in F_{3}(i=1,2)$.

TrEorem 2. Let $\beta$ be a real number. Then there exist four numbers $\alpha_{j} \in F_{2}(j=1,2,3,4)$ such that $\beta=\alpha_{1}+\alpha_{2}+\alpha_{3}+\alpha_{4}$. Also there exist real numbers $\beta^{\prime}$ such that $\beta^{\prime} \neq \alpha_{1}^{\prime}+\alpha_{2}^{\prime}+\alpha_{3}^{\prime}$ for any triple $\left(a_{3}^{\prime}, a_{2}^{\prime}, a_{3}^{\prime}\right), \alpha_{i}^{\prime} \in F_{2}$ $(i=1,2,3)$.

Now, let $k \geqslant 2$ be a natural number. Let $A_{1}, A_{2}, \ldots, A_{k}$ be non-empty sets of real numbers. We shall call their Schnirelman sum (notation $\left.A_{1}+A_{2}+\ldots+A_{k}\right)$ the set of all numbers of the form $\alpha_{1}+\alpha_{2}+\ldots+\alpha_{k}$, where $\alpha_{j} \in A_{j}(j=1,2, \ldots, k)$. Theorems 1 and 2 then follow from Theorems $1^{\prime}$ and $2^{\prime}$, respectively.

THEOREM $\mathcal{1}^{\prime}$.

THEOREM $2^{\prime}$

$$
\begin{gathered}
F_{3}+F_{3}+F_{3}=(-\infty,+\infty), \\
F_{3}+F_{3} \neq(-\infty,+\infty) .
\end{gathered}
$$

$$
\begin{gathered}
F_{2}+F_{2}+F_{2}+F_{2}=(-\infty,+\infty), \\
F_{2}+F_{2}+F_{2} \neq(-\infty,+\infty) .
\end{gathered}
$$


For integral numbers $k \geqslant 0$ and for natural numbers $N$ we denote by the symbol $F_{N}\left(n_{0}, n_{1}, \ldots, v_{b_{k}}\right)$ the set of all $a \in F_{N}, a=\left(g_{0} ; g_{1}, g_{2}, \ldots\right)$, for which $g_{i}=n_{i}(i=0,1, \ldots, k)$. Theorems $X^{\prime}$ and $2^{\prime}$ then follow from Theorems $1^{\prime \prime}$ and $2^{\prime \prime}$.

TTEOREM $1^{\prime \prime}$.

$$
F_{3}(0)+F_{3}(0)+F_{3}(0)=\left[\frac{\sqrt{21}-3}{2}, 3 \frac{\sqrt{21}-3}{2}\right],
$$

$$
F_{3}(0)+F_{3}(0) \subset\left[\frac{\sqrt{21}-3}{2}, \frac{15-\sqrt{21}}{17}\right] \cup\left[\frac{4 \sqrt{21}-9}{15}, \sqrt{21}-3\right] .
$$

Theorem $2^{\prime \prime}$.

$$
F_{2}(0)+F_{2}(0)+F_{2}(0)+F_{2}(0)=[2(\sqrt{3}-1), 4(\sqrt{3}-1)],
$$

(4.") $F_{2}(0)+F_{2}(0)+F_{2}(0) \subset\left[3 \frac{\sqrt{3}-1}{2}, 3-\sqrt{3}\right] \cup\left[\frac{4 \sqrt{3}-3}{3}, 3(\sqrt{3}-x)\right]$.

We have obviously

$$
\begin{aligned}
F_{3}+F_{3}+F_{3} & =\bigcup_{\substack{n_{0}, n_{0,}^{\prime}, n_{0}^{\prime \prime} \\
\text { int. }}}\left(F_{3}\left(n_{0}\right)+F_{3}\left(n_{0}^{\prime}\right)+F_{3}^{\prime}\left(n_{0}^{\prime \prime}\right)\right) \\
& =\bigcup_{\substack{n_{0}, n_{0,}^{\prime}, n_{0}^{\prime \prime} \\
\text { tnt. }}}\left(F_{3}(0)+F_{3}(0)+F_{3}(0)+\left\{n_{0}+n_{0}^{\prime}+n_{0}^{\prime \prime}\right\}\right\} \\
& =\bigcup_{n \text { tat. }}\left(F_{3}(0)+F_{3}(0)+F_{3}(0)+\{n\}\right)
\end{aligned}
$$

and the length of the interval $\left[\frac{\sqrt{21}-3}{2}, 3 \frac{\sqrt{21}-3}{2}\right]$ is $\sqrt{21}-3>1$. Hence, $\left(1^{\prime}\right)$ follows from $\left(1^{\prime \prime}\right)$. Similarly, we have

$$
F_{8}+F_{3}=\bigcup_{n \text { int. }}\left(H_{3}^{\prime}(0)+F_{3}(0)+\{n\}\right) .
$$

Since $\frac{4 \sqrt{21}-9}{15}>\frac{15-\sqrt{21}}{17}$,

$$
\begin{aligned}
& \max \left(F_{3}(0)+F_{3}(0)+\{-1\}\right)=\sqrt{21}-4<\frac{15-\sqrt{21}}{17} \\
& \min \left(F_{3}(0)+F_{3}(0)+\{1\}\right)=\frac{\sqrt{21}-3}{3}+1>\frac{4 \sqrt{21}-9}{15}
\end{aligned}
$$

the inclusion $\left(2^{\prime \prime}\right)$ implies $\left(2^{\prime}\right)$. In an analogous manner we get very ensily that $\left(3^{\prime \prime}\right)$ implies $\left(3^{\prime}\right)$.

Finally, since $\frac{4 \sqrt{3}-3}{3}>3-\sqrt{3}$

$$
\begin{aligned}
& \max \left(F_{2}(0)+F_{2}(0)+F_{2}(0)+\{-1\}\right)=3(\sqrt{3}-1)-1<3-\sqrt{3}, \\
& \min \left(F_{2}(0)+F_{2}(0)+F_{2}(0)+\{1\}\right)=3 \frac{\sqrt{3}-1}{2}+1>\frac{4 \sqrt{3}-3}{3},
\end{aligned}
$$

the inclusion $\left(4^{\prime \prime}\right)$ implies $\left(4^{\prime}\right)$.

We start with the proof of inclusions $\left(2^{\prime \prime}\right)$ and $\left(4^{\prime \prime}\right)$.

Proof of $\left(2^{\prime \prime}\right)$. We have obviously $F_{3}(0)=F_{3}(0,1) \cup F_{3}(0,2) \cup$ $\cup F_{3}(0,3)$ and also

$$
\begin{aligned}
F_{3}(0,3) \subset[(0 ; 3, \overline{1,3}),(0 ; 3, \overline{3,1})] & =\left[\frac{\sqrt{21}-3}{6}, \frac{15-\sqrt{21}}{34}\right] \stackrel{\text { df }}{=} K_{1}, \\
F_{3}(0,2) \cup F_{3}(0,1) \subset[(0 ; 2, \overline{1,3}),(0 ; 1, \overline{3,1})] & \\
= & {\left[\frac{\sqrt{21}-1}{10}, \frac{\sqrt{21}-3}{2}\right] \stackrel{\text { df }}{=} K_{2} . }
\end{aligned}
$$

From this follows that $F_{3}(0) \subset K_{1} \cup K_{2}$ and hence

$$
\begin{aligned}
F_{3}(0)+F_{3}(0) \subset\left(K_{1}+K_{1}\right) \cup\left(K_{1}+K_{2}\right) \cup\left(K_{2}+K_{2}\right) \\
=\left[\frac{\sqrt{21}-3}{3}, \frac{15-\sqrt{21}}{17}\right] \cup\left[\frac{4 \sqrt{21}-9}{15}, \sqrt{21}-3\right] .
\end{aligned}
$$

Proof of $\left(4^{\prime \prime}\right)$. Obviously, $F_{2}(0)=F_{2}(0,1) \cup F_{2}(0,2)$ and also

$$
\begin{gathered}
F_{2}(0,2) \subset[(0 ; 2, \overline{1,2}),(0 ; 2, \overline{2,1})]=\left[\frac{\sqrt{3}-1}{2}, \frac{3-\sqrt{3}}{3}\right] \stackrel{\text { df }}{=} K_{3}, \\
F_{2}(0,1) \subset[(0 ; 1, \overline{1,2}),(0 ; 1, \overline{2,1})]=\left[\frac{\sqrt{3}}{3}, \sqrt{3}-1\right] \stackrel{\text { df }}{=} K_{4} .
\end{gathered}
$$

From this follows that $F_{2}(0) \subset K_{3} \cup K_{4}$ and hence

$$
\begin{aligned}
& F_{2}^{\prime}(0)+F_{2}(0)+F_{2}^{\prime}(0) \\
& \subset\left(K_{3}+K_{3}+K_{3}\right) \cup\left(K_{3}+K_{3}+K_{4}\right) \cup\left(K_{3}+K_{4}+K_{4}\right) \cup\left(K_{4}+K_{4}+K_{4}\right) \\
& \quad=\left[3 \frac{\sqrt{3}-1}{2}, 3-\sqrt{3}\right] \cup\left[\frac{4 \sqrt{3}-3}{3}, 3(\sqrt{3}-1)\right] .
\end{aligned}
$$

We shall prove relations $\left(1^{\prime \prime}\right),\left(3^{\prime \prime}\right)$ as an application of the following more general considerations. 
Let $I_{0}$ be a non-degenerate compact interval with endpoints $a, b$, i.e. $I_{0}=[a, b]$. We sball call $I_{0}$ an interval of order 0 . We delete from $I_{0}$ an open interval $(c, d)$, such that $a<0<d<b$. We get thus two closed. intervals $I_{1}^{(1)}=[a, c], I_{1}^{(2)}=[d, b]$, which we shall call intervals of order 1. Generally, when we have already constructed all intervals $I_{n}^{\left(i_{1} \ldots i_{n}\right)}$ of order $n$ for some $n \geqslant 0$ we get intervals $I_{n+1}^{\left(i_{1} \ldots i_{n} 1\right)}, I_{n+1}^{\left(i_{1} \ldots i_{n}{ }^{2}\right)}$ of order $n+1$ by deleting from each interval of order $n$ an open non-empty interval, in such a way that the resulting intervals of order $n+1$ are non-degenorate. If we denote by $K_{n}(n \geqslant 0)$ the union of all intervals of order w, then $\bigcap_{n \geqslant 0} K_{n} \stackrel{d a}{=} L_{(}\left(X_{0}\right)$ is a closed, non-countable set contained in $I_{0}$ and with measure less than the length of $I_{0} . L\left(I_{0}\right)$ contains either. a non-emptiy interval or is nowhere dense in $I_{0}$.

In. the following, we shall denote by $\mu(A)$ the moasure (length) of an interval $A$.

DEFINITION. We shall say that $L\left(I_{0}\right)$ satisfies the ki-condition, if for arbitrary $n \geqslant 0$, the lengths of the intervals $I_{n}^{\left(i_{1} \ldots i_{n}\right)}, I_{n+1}^{\left(i_{1}, \ldots i_{n}, 1\right)}$ and $I_{n+1}^{\left(i_{1} \ldots i_{n} 2\right)}$ satisfy the following two inequalities:

$$
\begin{aligned}
& \mu\left(I_{n}^{\left(i_{1} \ldots i_{n}\right)}\right) \leqslant k \cdot \mu\left(I_{n+1}^{\left(i_{1} \ldots i_{n} 1\right)}\right)+\mu\left(I_{n+1}^{\left(i_{1} \ldots i_{n k} 2\right)}\right), \\
& \mu\left(I_{n}^{\left(i_{1} \ldots i_{n}\right)}\right) \leqslant \mu\left(I_{n+1}^{\left(i_{1} \ldots i_{n} 1\right)}\right)+k \cdot \mu\left(I_{n+1}^{\left(i_{1} \ldots i_{k} 2\right)}\right) .
\end{aligned}
$$

The following assertion, in a different formulation was proved by M. Hall Jr. [1].

ASSERTION 1. Let $A, B$ be nom-degenerate compact intervals and $L(A)$, $L(B)$ satisfy the 2 -condition. If $\frac{1}{3} \leqslant \mu(A) / \mu(B) \leqslant 3$, then

$$
L(A)+L(B)=A+B .
$$

For the proof of $\left(1^{\prime \prime}\right)$ we shall need an analogue of Assertion 1 for three "summands";

ASSERTION 2. Let $A, B, O$ be non-degenerate oompant internals and $L(A), \quad L(B), \quad L(C)$ satisfy the 3-condition. If $\mu(A)+\mu(B)+\mu(C) \leqslant$ $\leqslant 6 \min (\mu(A), \mu(B), \mu(O))$, then

$$
L(A)+L(B)+L(C)=A+B+C .
$$

For the proof of $\left(3^{\prime \prime}\right)$ we shall need an analogue of Assertion 1 for four "summands":

ASSERTION 3. Let $A, B, C, D$ be non-degenerate compact intervals and $L(A), L(B), L(C), L(D)$ satisfy the 4-condition. If $\mu(A)+\mu(B)+\mu(O)+$ $+\mu(D) \leqslant 8 \min (\mu(A), \mu(B), \mu(C), \mu(D))$, then

$$
L(A)+L(B)+L(O)+L(D)=A+B+O+D .
$$

Remark. The similarities of Assertions 2 and 3 with Assertion 1 will become clearer, when we rewrite the condition $\frac{1}{3} \leqslant \mu(A) / \mu(B) \leqslant 3$ from Assertion 1 to the equivalent form $\mu(A)+\mu(B) \leqslant 4 \min (\mu(A), \mu(B))$.

Instead of Assertions 2 and 3 we shall formulate and prove more general Assertions $2^{\prime}$ and $3^{\prime}$.

ASSERTION $2^{\prime}$. Let $A=\left[A_{L}, A_{R}\right], B=\left[B_{L}, B_{R}\right], C=\left[C_{L}, C_{R}\right]$ be non-degenerate compact intervals. Let $L(A), L(B), L(C)$ satisfy the 3-condition. Then

$$
\begin{aligned}
{\left[A_{L}+B_{L}+C_{L}, A_{L}+B_{L}+C_{L}+3 \min (\mu(A), \mu(B), \mu(C))\right] } & \\
& \subset L(A)+L(B)+L(C) \\
{\left[A_{R}+B_{R}+C_{R}-3 \min (\mu(A), \mu(B), \mu(C)), A_{R}+B_{R}+O_{R}\right] } & \subset L(A)+L(B)+L(O) .
\end{aligned}
$$

Assertion $3^{\prime}$. Let $A=\left[A_{L}, A_{R}\right], B=\left[B_{L}, B_{R}\right], C=\left[O_{L}, C_{R}\right]$, $D=\left[D_{L}, D_{R}\right]$ be non-degenerate compact intervals. Let $L(A), L(B), L(C)$, $L(D)$ satisfy the 4-condition. Then

$$
\begin{aligned}
& {\left[A_{L}+B_{L}+O_{L}+D_{L}, A_{L}+B_{L}+C_{L}+D_{L}+4 \min (\mu(A), \mu(B), \mu(C), \mu(D))\right] } \\
& \simeq L(A)+L(B)+L(C)+L(D), \\
& {\left[A_{R}+B_{R}+C_{R}+D_{R}-4 \min (\mu(A), \mu(B), \mu(C), \mu(D)), A_{R}+B_{R}+C_{R}+D_{R}\right] } \\
& \subset L(A)+L(B)+L(C)+L(D) .
\end{aligned}
$$

The proofs of Assertions $2^{\prime}$ and $3^{\prime}$ will make use of Lemmas 1 and 2 below. Before proceeding, some new definitions are needed.

Defintion. Let $A_{j}=\left[x_{j}, x_{j}+a_{j}\right](j=1,2, \ldots, n)$ be $n(n \geqslant 2)$ nondegenerate compact intervals. The interval

$$
\underline{\left(A_{1}, A_{2}, \ldots, A_{n}\right)}=\left[\sum_{j=1}^{n} x_{j}, \sum_{j=1}^{n} x_{j}+n \min _{1 \leqslant j \leqslant n} a_{j}\right]
$$

will be called their lower associate, the interval

$$
\left.\overline{\left(A_{1}, A_{2}, \ldots, A_{n}\right.}\right)=\left[\sum_{j=1}^{n}\left(x_{j}+a_{j}\right)-n \min _{1 \leqslant j \leqslant n} a_{j}, \sum_{j=1}^{n}\left(x_{j}+a_{j}\right)\right]
$$

will be called their upper associate.

Lemma 1. Let $x_{j}(j=1,2,3)$ be real numbers: Let $a_{j}(j=1,2,3), b_{1}, c_{1}$ be positive numbers, such that $a_{1} \geqslant a_{2} \geqslant a_{3}, a_{1}>b_{1}+e_{1}$,

$$
\begin{aligned}
& a_{1} \leqslant b_{1}+3 c_{1}, \\
& a_{1} \leqslant 3 b_{1}+c_{1} .
\end{aligned}
$$


Let us put

$$
\begin{aligned}
A_{j} & =\left[x_{j}, x_{j}+a_{j}\right] \quad(j=1,2,3) \\
A_{11} & =\left[x_{1}, x_{1}+b_{1}\right], \\
A_{12} & =\left[x_{1}+a_{1}-c_{1}, x_{1}+a_{1}\right] .
\end{aligned}
$$

Then

(i) $\left(A_{1}, A_{2}, A_{3}\right) \cup\left(\overline{A_{1}, A_{2}, A_{3}}\right) \subset \bigcup_{j=1}^{2}\left(\left(A_{1 j}, \dot{A_{2}}, A_{3}\right) \cup\left(A_{1 j}, A_{2}, A_{3}\right)\right)$.

Proof. Because the situation is completely iflvariant with respect to the numbers $x_{j}(j=1,2,3)$, we may restrict ourselves to the case $x_{1}=x_{2}=x_{3}=0$. Moreover, it suffices to prove the inclusion (7) only for $\left(A_{1}, A_{2}, A_{3}\right)$ due to the symmetry between the letters $b, o$ and the lower and upper associates.

By the very definition and by the assumptions of the lemma we have

$$
\left(\underline{A_{1}, A_{2}, A_{3}}\right)=\left[0,3 a_{3}\right], \quad\left(A_{11}, A_{2}, A_{3}\right)=\left[0,3 \min \left(a_{3}, b_{1}\right)\right] .
$$

Hence, if $b_{1} \geqslant a_{3}$, we are done. Let in the following $b_{1}<a_{3}$, i.e. $\left(A_{11}, A_{2}, A_{9}\right)$ $=\left[0,3 b_{1}\right]$. We have

$$
\underline{\left(A_{12}, A_{2}, A_{3}\right)}=\left[a_{1}-c_{1}, a_{1}-c_{1}+3 \min \left(c_{1}, a_{3}\right)\right]
$$

and using $(6)$ :

$$
\left[0, a_{1}-c_{1}+3 \min \left(c_{1}, a_{3}\right)\right] \subset\left(\underline{\left.A_{11}, A_{2}, A_{3}\right)} \cup\left(\underline{A_{12}, A_{2}, A_{3}}\right) .\right.
$$

Hence, if $c_{1} \geqslant a_{3}$, we are done. Tet in the following also $o_{1}<a_{3}$, i.e.

$$
\left[0, a_{1}+2 e_{1}\right] \subset\left(A_{11}, A_{2}, A_{3}\right) \cup\left(\underline{A_{12}, A_{2}, A_{3}}\right) .
$$

Now, we have

$$
\begin{gathered}
\left(\overline{A_{11}, A_{2}, A_{3}}\right)=\left[a_{2}+a_{3}-2 b_{1}, a_{2}+a_{3}+b_{1}\right], \\
\left(\overline{\left.A_{12}, A_{2}, A_{3}\right)}=\left[a_{1}+a_{2}+a_{3}-3 a_{1}, a_{1}+a_{2}+a_{3}\right] .\right.
\end{gathered}
$$

Using (5), we get

(9) $\left[a_{2}+a_{3}-2 b_{1}, a_{1}+a_{2}+a_{3}\right] \subset\left(\overrightarrow{A_{11}}, A_{2}, A_{3}\right) \cup\left(A_{12}, A_{2}, A_{3}\right)$.

From the relations (8), (9) we obtain that

$$
\left[0, a_{1}+a_{2}+a_{3}\right] \subset \bigcup_{j=1}^{2}\left(\left(A_{1 j}, A_{2}, A_{3}\right) \cup\left(A_{1 j}, A_{2}, A_{3}\right)\right),
$$

since $a_{2}+a_{3}-2 b_{1} \leqslant 2 a_{1}-2 b_{1} \leqslant a_{1}+2 c_{1}$ by $(5),(6)$.
LEMMA 2. Let $x_{j}(j=1,2,3,4)$ be real numbers. Let $a_{j}(j=1,2,3,4)$, $b_{j}, o_{j}(j=1,2,3)$ be positive numbers such that

Let us denote

$$
\begin{gathered}
a_{4}=\min _{1 \leqslant j \leqslant 4} a_{j}, \\
a_{j}>b_{j}+c_{j} \quad(j=1,2,3), \\
a_{j} \leqslant 4 b_{j}+c_{j} \quad(j=1,2,3), \\
a_{j} \leqslant b_{j}+4 c_{j} \quad(j=1,2,3) .
\end{gathered}
$$

$$
\begin{array}{rlrl}
A_{j 0} & =\left[x_{j}, x_{j}+a_{j}\right] & & (j=1,2,3,4), \\
A_{j 1} & =\left[x_{j}, x_{j}+b_{j}\right] & & (j=1,2,3), \\
A_{j 2} & =\left[x_{j}+a_{j}-c_{j}, x_{j}+a_{j}\right] & (j=1,2,3) .
\end{array}
$$

Then

(14) $\quad\left(A_{10}, A_{20}, A_{30}, A_{10}\right) \cup\left(\overline{A_{10}, A_{20}, A_{30}, \overline{A_{40}}}\right)$

$$
\subset \underset{\substack{0 \leqslant j, k, l \leqslant 2 \\ j+k+l \geqslant 1}}{\bigcup}\left(\left(A_{1 j}, A_{2 k}, A_{3 l}, A_{40}\right) \cup\left(\overline{A_{1 j}, A_{2 k}, A_{3 l}, A_{40}}\right)\right) .
$$

Proof. For the same reasons as in the proof of Lemma 1 we shall restrict ourselves only to the proof of the inclusion (14) for the lower associate $\left(\dot{A}_{10}, A_{20}, A_{30}, A_{40}\right)$ and, without loss of generality, we shall suppose that $x_{1}=x_{2}=x_{3}=x_{4}=0$. Hence we have

If $b_{1} \geqslant a_{4}$, then

$$
\left(\underline{\left.A_{10}, A_{20}, A_{80}, A_{40}\right)}=\left[0,4 a_{4}\right]\right. \text {. }
$$

$$
\left(A_{11}, A_{20}, A_{30}, A_{40}\right)=\left[0,4 a_{4}\right]
$$

and we are done. The cases $b_{2} \geqslant a_{4}, b_{3} \geqslant a_{4}$ can be handled similarly. Let in the following

$$
\max _{1<j<3} b_{j}<a_{4}
$$

which means that

$$
\text { (16) } \frac{\left(A_{11}, A_{20}, A_{30}, A_{40}\right)}{=} \frac{\left(A_{10}, A_{21}, A_{30}, A_{40}\right) \cup\left(A_{10}, A_{20}, A_{31}, A_{40}\right)}{=\left[0, \underset{1 \leqslant j \leqslant 3}{\left.4 \max _{j}\right]} .\right.}
$$

Now, $\left(A_{12}, A_{20}, A_{30}, A_{40}\right)=\left[a_{1}-c_{1}, a_{1}-c_{1}+4 \min \left(c_{1}, a_{4}\right)\right]$ and by (12), this interval overlaps the interval $(16)$. If $c_{1} \geqslant a_{4}$, then we are done and also in the cases $c_{2} \geqslant a_{4}$ or $c_{3} \geqslant a_{4}$, by an analogous argument. Hence, we shall suppose in the following that 
It follows from the considerations above, the lower associates which occur on the right-hand side of the inclusion (14), cover the interval

$$
\left[0, \max \left\{\max _{1 \leqslant j \leqslant 3} 4 b_{j} ; \max _{1 \in j \leqslant 3}\left(a_{j}+3 c_{j}\right)\right\}\right] \text {. }
$$

Completely analogously we could show, that the upper associates which occur on the right-lhand side of the inclusion (14), cover the interval

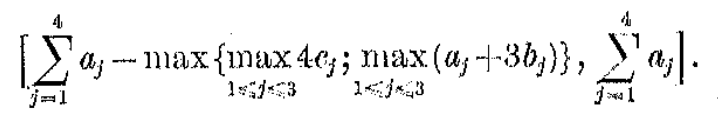

Unfortunately, in the general case, the union of the intervals (18) and (19) does not cover the interval $\left[0,4 a_{A}\right]$; however, fortunately, we still have not used all the associates on the right-hand side of the inclusion. (14).

We shall show that the interval $\left(A_{12}, A_{22}, A_{32}, A_{40}\right)$ overlaps the interval (19). This statement is equivalent to the inequality

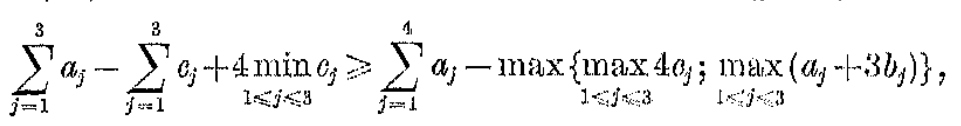

which is equivalent to

$$
\text { (20) } \quad \max \left\{\max _{1 \leqslant j \leqslant 3} 4 a_{j} ; \max _{1 \leqslant j \leqslant 3}\left(a_{j}+3 b_{j}\right)\right\}+4 \min _{1 \leqslant j} o_{j}>a_{4}+\sum_{j=1}^{3} e_{j} .
$$

The last inequality can be easily proved using the relutions (10), (12) and (1,3). Namely, we have

$$
\begin{aligned}
& \max \left\{\max 4 c_{j} ; \max \left(a_{j}+3 b_{j}\right)\right\}+4 \min c_{j} \\
& \geqslant \frac{1}{2} \max _{1 \leqslant j \leqslant 3} 4 c_{j}+\frac{1}{2} \max _{1 \leqslant j \leqslant 3}\left(a_{j}+3 b_{j}\right)+4 \min _{1 \leqslant j \leqslant 3} c_{j}
\end{aligned}
$$

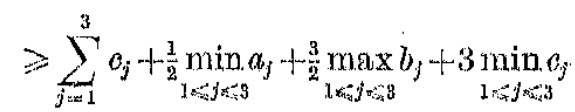

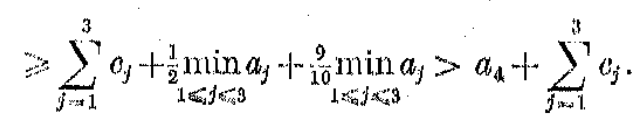

Quite analogously we could show that the interval $\left(A_{11}, A_{2,1}, A_{8,1}, A_{4(1)}\right)$ overlaps the interval (18). Hence, under the assumptions (15) and (17), the following intervals will certainly be covered:

$$
\begin{gathered}
{\left[0, \max \left\{\max _{1 \leqslant j \leqslant 3} 4 b_{j} ; \max _{1 \leqslant j \leqslant 3}\left(a_{j}+3 c_{j}\right) ; a_{4}+\sum_{j=1}^{3} b_{j}\right\}\right],} \\
{\left[\sum_{j=1}^{4} a_{j}-\max \left\{\max _{1 \leqslant j \leqslant 3} 4 c_{j} ; \max _{1 \leqslant j \leqslant 3}\left(a_{j}+3 b_{j}\right) ; a_{4}+\sum_{j=2}^{3} c_{j}\right\}, \sum_{j=1}^{4} a_{j}\right] .}
\end{gathered}
$$

Unfortunately, even the union of the intervals $(21),(22)$ need not cover the interval $\left[0,4 a_{4}\right]$. But we still have not made use of all the intervals on the right-hand side of the inclusion (14). Moreover, we shall show that the whole interval $\left[0, \sum_{j=1}^{4} a_{j}\right]$ is covered.

Oase A. Let

$$
b_{j} \leqslant c_{j}(j=1,2,3) \text { and } c_{1} \geqslant c_{2} \geqslant c_{8} .
$$

We show that the interval $\left(A_{12}, A_{22}, A_{30}, A_{40}\right)$ overlaps the interval (21). If not, we have

$$
a_{1}-c_{1}+a_{2}-c_{2}>a_{1}+3 c_{1}, \quad a_{1}-c_{1}+a_{2}-c_{2}>a_{2}+3 c_{2} .
$$

Adding the inequalities (24) we would get

$$
a_{1}+a_{2}>5 a_{1}+5 c_{2} \geqslant c_{1}+4 b_{1}+c_{2}+4 b_{2},
$$

contradicting the assumptions (12), (13). Similarly we get that also the intervals $\left(A_{12}, A_{20}, A_{32}, A_{40}\right),\left(A_{10}, A_{22}, A_{32}, A_{40}\right)$ overlap the interval (21). Hence, certainly the interval

$$
\left[0, \max \left\{a_{1}-c_{1}+a_{2}+3 c_{2} ; a_{1}-c_{1}+a_{3}+3 c_{3}\right\}\right]
$$

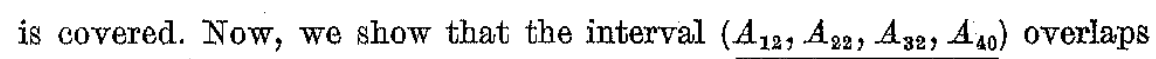
(25). If not, we have

$$
\begin{aligned}
& a_{1}-c_{1}+a_{2}-c_{2}+a_{3}-c_{3}>a_{1}-c_{1}+a_{2}+3 c_{2}, \\
& a_{1}-c_{1}+a_{2}-c_{2}+a_{3}-c_{3}>a_{1}-c_{1}+a_{3}+3 c_{3} .
\end{aligned}
$$

Adding the inequalities (26) we would get

$$
a_{2}+a_{3}>5 c_{2}+5 c_{3} \geqslant c_{2}+4 b_{2}+c_{3}+4 b_{3}
$$

thus contradicting (12) and (13). Now; the interval $\left(\underline{A}_{12}, A_{22}, A_{22}, A_{40}\right)$ is contained in (22) and hence we have shown that (25) overlaps (22), i.e. the whole interval $\left[0, \sum_{j=1}^{4} a_{j}\right]$ is covered.

Case B. Let

$$
b_{j} \leqslant c_{j}(j=1,2), \quad b_{3}>c_{3}, \quad c_{1} \geqslant c_{2} .
$$

Again as in case $\mathrm{A}$, the interval $\left(A_{12}, A_{22}, A_{30}, A_{40}\right)$ overlaps the interval (21). We shall distinguish three subcases.

Subcase B1. Let

$$
b_{3} \geqslant c_{1}
$$


Then the interval $\left(A_{12}, A_{22}, A_{30}, A_{40}\right)$ overlaps the interval (22), since (by (27), (28), (12), (13) and (10))

$$
\begin{aligned}
a_{1}-c_{1}+a_{2}+3 c_{2} & \geqslant a_{1}+a_{2}+2 e_{1}+3 c_{2}-3 b_{3} \geqslant a_{1}+a_{2}+2 b_{2}+3 c_{2}-3 b_{3} \\
& \geqslant a_{1}+a_{2}+a_{4}-3 b_{3}=\sum_{j=1}^{4} a_{j}-\left(a_{3}+3 b_{3}\right) .
\end{aligned}
$$

Thus the whole interval $\left[0, \sum_{j=1}^{4} a_{j}\right]$ is covered.

$$
b_{3}<c_{1}, \quad 4 c_{2}+c_{3} \geqslant a_{3} .
$$

Then the interval $\left(A_{12}, A_{22}, A_{30}, A_{40}\right)$ overlaps the interval (22), since (by (29))

$$
a_{1}-e_{1}+a_{2}+3 c_{2} \geqslant a_{1}-e_{1}+a_{2}-c_{2}+a_{3}-c_{3}=\sum_{j=1}^{4} a_{j}-\left(a_{4}+\sum_{j=1}^{3} o_{j}\right) .
$$

Thus the whole interval $\left[0, \sum_{j=1}^{i} a_{j}\right]$ is covered.

Subcase B3. Let

$$
b_{3}<e_{1}, \quad 4 c_{2}+c_{3}<a_{3} .
$$

The assumption $4 c_{2}+e_{3}<a_{3}$ implies (using (12))

thus

$$
4 e_{2}+e_{3}<a_{\mathrm{s}} \leqslant 4 b_{\mathrm{a}}+c_{3},
$$

hence (using (27))

(32)

thus (by (13), (31))

$$
\begin{aligned}
& c_{2}<b_{3}, \\
& b_{2}<b_{3},
\end{aligned}
$$$$
a_{2} \leqslant b_{2}+4 c_{2}<b_{2}+4 b_{8} .
$$

We shall show that the interval $\left(A_{10}, A_{21}, A_{31}, A_{40}\right)$ overlaps the interval

(21). We have (using (32), (10), (30), (12), (13), (27))

$$
\begin{aligned}
\sum_{j=1}^{4} a_{j}-\left(a_{2}-b_{2}+a_{3}-b_{3}+4 \underset{j=2,3}{\min } b_{j}\right)=a_{1}+a_{4}+b_{3}-3 b_{2} \\
\leqslant a_{1}+a_{2}+o_{1}-3 b_{2} \leqslant a_{1}+a_{1}+2 o_{2} \leqslant a_{1}+3 o_{1} .
\end{aligned}
$$

Finally, we show that the interval $\left(\overline{A_{10}}, A_{21}, A_{31}, A_{40}\right)$ overlaps the interval (22). We have (by (33))

$$
\sum_{j=1}^{4} a_{j}-\left(a_{2}-b_{2}+a_{3}-b_{3}\right) \geqslant \sum_{j=1}^{4} a_{j}-\left(a_{3}+3 b_{3}\right) .
$$

Thus again the whole interval $\left[0, \sum_{j=1}^{4} a_{j}\right]$ is covered.
Case $O$. We have neither case A nor B. Then by a suitable renumbering or change of the role of the letters $b$ and $c$, we can reduce the situation to one of the cases $A, B$.

Proof of Assertion 2'. The intervals on the left-hand sides of the inclusions in Assertion $2^{\prime}$ are associates $(A, B, C)$ and $(\overline{A, B, C})$. If a number $\delta$ belongs to one of them, then (by Lemma 1) $\delta$ belongs to an infinite sequence of nested closed associates $\left(A^{(i)}, B^{(i)}, O^{(i)}\right)(i=1,2, \ldots)$ (upper or lower) such that $A^{(i+1)} \subset A^{(i)}, B^{(i+1)} \subset B^{(i)}, C^{(i+1)} \subset C^{(i)}(i=1,2, \ldots)$ and we cannot have equality in all three cases. Also, $A^{(1)}=A, B^{(1)}=B$, $C^{(1)}=C$ and each of the intervals $A^{(i)}$ or $B^{(i)}$ or $C^{(i)}$ contains points of $L(A)$ or $L(B)$ or $L(C)$, respectively. If the sets $L(A), L(B)$ and $L(C)$ do not contain any interval, then, since 1 ) the intervals $A^{(i)}, B^{(i)}, O^{(i)}$ are closed, 2) always the shortest one of them remains undivided at each step (unless they have the same length), 3 ) the associates $\left(A^{(i)}, B^{(i)}, O^{(i)}\right.$ ) are closed, 4) $\left(A^{(i)}, B^{(i)}, C^{(i)}\right) \subset A^{(i)}+B^{(i)}+C^{(i)}(i=1,2,3, \ldots)$, the intersections $\bigcap_{i} A^{(i)}, \bigcap_{i} B^{(i)}, \bigcap_{i} C^{(i)}$ contain just one point and we have

$$
\begin{gathered}
\bigcap_{i} A^{(i)}=\{\alpha\} \subset L(A), \quad \bigcap_{i} B^{(i)}=\{\beta\} \subset L(B), \quad \bigcap_{i} C^{(i)}=\{\gamma\} \subset L(C), \\
\alpha+\beta+\gamma=\delta, \quad\{\delta\}=\bigcap_{i}\left(A^{(i)}, B^{(i)}, C^{(i)}\right) .
\end{gathered}
$$

If some of the sets $L(A), L(B), L(C)$ contains an interval, e.g. $L(A)$ say, then we can delete from $L(A)$ countably many open intervals such that the resulting set $L^{\prime}(A)$ does not contain any interval and such that, by a suitable ordering of the steps in the construction of the set $L^{\prime}(A)$ from the interval $A$ by the scheme above, the 3 -condition will be satisfied. This can be achieved easily e.g. so, that from each interval contained in $L(A)$, we delete countably many open intervals in a manner analogous to the construction of the Cantor discontinuum (as far as the ratios of the lengths of the corresponding intervals are eoncerned). Then we construct the set $L^{\prime}(A)$ in the following way. We shall delete from the interval $A$ all the intervals which have been deleted by the construction of the set $L(A)$ and all the intervals which were deleted from the intervals contained in the set $L(A)$ in such an order, that at each step we delete from each interval to be divided a longest possible to be deleted.

In the same manner we construct the sets $L^{\prime}(B), L^{\prime}(C)$. If $L(B)$ does not contain any interval, then, of course, $L^{\prime}(B)=L(B)$; similarly in the case of $L^{\prime}(C)$. From the first part of this proof then follows that $\delta=\alpha^{\prime}+\beta^{\prime}+\gamma^{\prime}$, where $\alpha^{\prime} \in L^{\prime}(A) \subset L(A), \quad \beta^{\prime} \in L^{\prime}(B) \subset L(B), \quad \gamma^{\prime} \in L^{\prime}(O)$. $\subset L(C)$.

Proof of Assertion $3^{\prime}$. The proof is completely analogous to the proof of Assertion 2'. 
Proof of $\left(1^{\prime \prime}\right)$ from Theorem $1^{\prime \prime}$. We shall show that

$$
F_{3}(0)=L\left(\left[\frac{\sqrt{21}-3}{6}, \frac{\sqrt{21}-3}{2}\right]\right)
$$

and $F_{3}(0)$ satisfies the 3 -condition.

We put $I_{0}=\left[\frac{1}{6}(\sqrt{21}-3), \frac{1}{2}(\sqrt{21}-3)\right]=[(0 ; \overline{3,1}),(0 ; 1,3)]$. From $I_{0}$ we delete the open interval $((0 ; 2, \overline{3,1}),(0 ; 1, \overline{1,3}))$, whose endpoints belong to the set $F_{3}(0)$, but which itself contains no point of $F_{3}(0)$. Wo get two closed intervals $I_{1}^{(1)}=[(0 ; 3, \overline{1,3}),(0 ; 2, \overline{3,1})], I_{1}^{(2)}=[(0 ; 1,1, \overline{3})$, $(0 ; 1, \overline{3,1})]$, such that their union covers $F_{3}^{\prime}(0)$. From the interval $X_{1}^{(1)}$ we delete the open interval $((0 ; 3, \overline{3,1}),(0 ; 2, \overline{1}, 3))$, from the interval $I_{2}^{(2)}$ we delete the open interval $(\langle 0 ; 1,1, \overline{1,3}),(0 ; 1,2, \overline{3,1}))$. Thus wo get four closed intervals

$$
\begin{aligned}
& I_{2}^{(2,1)}=[(0 ; 1,1, \overline{3,1}),(0 ; 1,1, \overline{1,3})], \\
& I_{2}^{(2,2)}=[(0 ; 1,2, \overline{3,1}),(0 ; 1,3, \overline{1,3})],
\end{aligned}
$$$$
I_{2}^{(1,1)}=[(0 ; 3, \overline{1,3}),(0 ; 3, \overline{3,1})], \quad I_{2}^{(1,2)}=[(0 ; 2, \overline{1}, \overline{3}),(0 ; 2, \overline{3}, \overline{1})]
$$

whose endpoints belong to $F_{3}(0)$ and such that the union covers $F_{3}(0)$. We show generally that anyone of the intervals $I_{n}^{\left(i_{1} \ldots i_{n}\right)}$ will have endpoints of one of the following two types:

$$
\begin{array}{rc}
\left(0 ; a_{1}, \ldots, a_{r}, \overline{1,3}\right), & \left(0 ; a_{1}, \ldots, a_{r}, \overline{3,1}\right), \\
\left(0 ; a_{1}, \ldots, a_{r}, 3, \overline{1,3}\right), & \left(0 ; a_{1}, \ldots, a_{r}, 2, \overline{3,1}\right) .
\end{array}
$$

Here, of course, the points on the left can be both left and right endpoints; this depends on the parity of $r$. We remark first of all that $I_{0}$ has endpoints of the type (34) (with $r=0$ ). When we have an interval with endpoints of the type (34), then after deleting the open interval with endpoints $\left(0 ; a_{1}, \ldots, a_{r}, 1, \overline{1,3}\right),\left(0 ; a_{1}, \ldots, a_{n}, 2, \overline{3,1}\right)$ wo get two closed intervals, the one with endpoints $\left(0 ; a_{1}, \ldots, a_{r}, 1, \overline{3,1}\right),\left(0 ; a_{1}, \ldots, a_{r}, 1,1,3\right)$ (of: type $(34))$, the other with endpoints $\left(0 ; a_{1}, \ldots, a_{r}, 2,3, \mathrm{~J}_{1}\right), \quad\left(0 ; a_{1}, \ldots\right.$ $\ldots, a_{r}, 3, \overline{1,3}$ ) (of type (35)). When we have an interval with endpoints of the type (35), then after deleting the open interval with endpointis $\left(0 ; a_{1}, \ldots, a_{r}, 3, \overline{3,1}\right),\left(0 ; a_{1}, \ldots, a_{r}, 2, \overline{1,3}\right)$ we get two closed intervals, the one with endpoints $\left(0 ; a_{1}, \ldots, a_{r}, 3, \overline{1,3}\right),\left(0 ; a_{1}, \ldots, a_{r}, 3, \overline{3,1}\right)$ (of type (34)), the other with endpoints $\left(0 ; a_{1}, \ldots, a_{r}, 2, \overline{1,3}\right),\left(0 ; a_{1}, \ldots\right.$ $\ldots, a_{r}, 2, \overline{3,1}$ ) (of type $\left.(34)\right)$. It is easily seen that by this process we get from the interval $\left[\frac{1}{6}(\sqrt{21}-3), \frac{1}{2}(\sqrt{21}-3)\right]$ just the set $F_{3}(0)$.
Case A. The division of an interval with endpoints of type (34). We must prove the following:

$$
\begin{aligned}
&\left|\left(0 ; a_{1}, \ldots, a_{r}, \overline{1,3}\right)-\left(0 ; a_{1}, \ldots, a_{r}, \overline{3,1}\right)\right| \\
& \leqslant\left|\left(0 ; a_{1}, \ldots, a_{r}, \overline{1}, \overline{3,1}\right)-\left(0 ; a_{1}, \ldots, a_{r}, 1, \overline{1}, 3\right)\right|+ \\
&+3\left|\left(0 ; a_{1}, \ldots, a_{r}, 2, \overline{3,1}\right)-\left(0 ; a_{1}, \ldots, a_{r}, 3, \overline{1,3}\right)\right| \\
&\left|\left(0 ; a_{1}, \ldots, a_{r}, \overline{1,3}\right)-\left(0 ; a_{1}, \ldots, a_{r}, \overline{3,1}\right)\right| \\
& \leqslant 3\left|\left(0 ; a_{1}, \ldots, a_{r}, 1, \overline{3,1}\right)-\left(0 ; a_{1}, \ldots, a_{r}, 1, \overline{1,3}\right)\right|+ \\
&+\left|\left(0 ; a_{1}, \ldots, a_{r}, 2, \overline{3,1}\right)-\left(0 ; a_{1}, \ldots, a_{r}, 3, \overline{1,3}\right)\right|
\end{aligned}
$$

The inequalities (36) and (37), respectively, are easily seen to be equivalent to the following inequalities, $\left(36^{\prime}\right)$ and $\left(37^{\prime}\right)$, respectively.

$$
\begin{aligned}
& \left|\frac{\left(0 ; a_{1}, \ldots, a_{r}, 1, \overline{1,3}\right)-\left(0 ; a_{1}, \ldots, a_{r}, 2, \overline{3,1}\right)}{\left(0 ; a_{1}, \ldots, a_{r}, 2, \overline{3,1}\right)-\left(0 ; a_{1}, \ldots, a_{r}, 3, \overline{1,3}\right)}\right| \leqslant 2, \\
& \left|\frac{\left(0 ; a_{1}, \ldots, a_{r}, 1, \overline{1}, 3\right)-\left(0 ; a_{1}, \ldots, a_{r}, 2, \overline{3,1}\right)}{\left(0 ; a_{1}, \ldots, a_{r}, 1, \overline{3}, 1\right)-\left(0 ; a_{1}, \ldots, a_{r}, 1, \overline{1,3}\right)}\right| \leqslant 2 .
\end{aligned}
$$

In the following, we shall denote by $x_{r-1} / y_{r-1}, x_{r} / y_{r}$ the last two convergents of the number $\left(0 ; a_{1}, \ldots, a_{r}\right)$ (for $r=0$ we set $x_{r-1}=1, y_{r-1}=0$ ). As well known, we have

$$
\text { (38) } \quad\left|x_{r} y_{r-1}-x_{r-1} y_{r}\right|=1 \text {. }
$$

We shall put

$$
\xi=\overline{(1 ; 3)}=\frac{\sqrt{21}+3}{6},
$$

so that $(\overline{3 ; 1})=3 \xi, 3 \xi^{2}=3 \xi+1 ;$ and

$$
t=\frac{y_{r-1}}{y_{r}}
$$

so that $0 \leqslant t \leqslant 1$.

Each of the continued fractions occurring on the left-hand sides of $\left.\left(36^{\prime}\right), 37^{\prime}\right)$ can be expressed in terms of $x_{r-1}, x_{r}, y_{r-1}, y_{r}, \xi$. We have

$$
\begin{aligned}
& \left(0 ; a_{1}, \ldots, a_{r}, 1, \overline{1,3)}=\frac{(3 \xi-2) x_{r}+x_{r-1}}{(3 \xi-2) y_{r}+y_{r-1}},\right. \\
& \left(0 ; a_{1}, \ldots, a_{r}, 2, \overline{3,1)}=\frac{(\xi+1) x_{r}+x_{r-1}}{(\xi+1) y_{r}+y_{r-1}},\right. \\
& \left(0 ; a_{1}, \ldots, a_{r}, 3, \overline{1,3}\right)=\frac{3 \xi x_{r}+x_{r-1}}{3 \xi y_{r}+y_{r-1}}, \\
& \left(0 ; a_{1}, \ldots, a_{r}, 1, \overline{3,1}\right)=\frac{\xi x_{r}+x_{r-1}}{\xi y_{r}+y_{r-1}} .
\end{aligned}
$$


When we substitute these expressions into the inequalities $\left(36^{\prime}\right),\left(37^{\prime}\right)$, we can easily see (using (38)) that the relations $\left(36^{\prime}\right),\left(37^{\prime}\right)$ are equivalent to the following inequalities:

$$
\begin{aligned}
& \frac{3-2 \xi}{2 \xi-1} \frac{3 \xi+t}{3 \xi-2+t} \leqslant 2, \\
& \frac{3-2 \xi}{2 \xi-2} \frac{\xi+t}{\xi+1+t} \leqslant 2 .
\end{aligned}
$$

Since $0 \leqslant t \leqslant 1$, the expression on the left-hand side of $\left(36^{\prime \prime}\right)$ is at most

$$
\frac{3-2 \xi}{2 \xi-1} \frac{3 \xi}{3 \xi-2}=\frac{\sqrt{21}}{7}<2 \text {. }
$$

Similarly, the expression on the left-hand side of $\left(37^{\prime \prime}\right)$ is at most

$$
\frac{3-2 \xi}{2 \xi-2} \frac{\xi+1}{\xi+2}=\frac{9 \sqrt{21}+1}{68}<2
$$

Case B. The division of an interval with endpoints of type (35). As in case $A$, it is sufficient to show that the following inequalities are satisfied:

$$
\begin{aligned}
& \left|\frac{\left(0 ; a_{1}, \ldots, a_{r}, 3, \overline{3,1}\right)-\left(0 ; a_{1}, \ldots, a_{r}, 2, \overline{1,3}\right)}{\left(0 ; a_{1}, \ldots, a_{r}, 3, \overline{1,3}\right)-\left(0 ; a_{1}, \ldots, a_{r}, 3, \overline{3,1}\right)}\right| \leqslant 2 \\
& \left|\frac{\left(0 ; a_{1}, \ldots, a_{r}, 3, \overline{3,1}\right)-\left(0 ; a_{1}, \ldots, a_{r}, 2, \overline{1,3}\right)}{\left(0 ; a_{1}, \ldots, a_{r}, 2, \overline{1,3}\right)-\left(0 ; a_{1}, \ldots, a_{r}, 2, \overline{3,1}\right)}\right| \leqslant 2 .
\end{aligned}
$$

Using the same notation as in case $\mathrm{A}$, we have:

$$
\begin{aligned}
& \left(0 ; a_{1}, \ldots, a_{r}, 2, \overline{1,3}\right)=\frac{(3 \xi-1) x_{r}+x_{r-1}}{(3 \xi-1) y_{r}+y_{r-1}}, \\
& \left(0 ; a_{1}, \ldots, a_{r}, 3, \overline{3,1}\right)=\frac{(\xi+2) x_{r}+x_{r-1}}{(\xi+2) y_{r}+y_{r-1}} .
\end{aligned}
$$

After using (41), (44) and (38) in (42) and (43), we see easily that the inequalities $(42),(43)$ are equivalent to the inequalities

$$
\begin{aligned}
& \frac{3-2 \xi}{2 \xi-2} \frac{\xi+2+t}{3 \xi-1+t} \leqslant 2, \\
& \frac{3-2 \xi}{2 \xi-2} \frac{\xi+1+t}{\xi+2+t} \leqslant 2 .
\end{aligned}
$$

Since $0 \leqslant t \leqslant 1$, the expression on the left-hand side of $\left(42^{\prime}\right)$ is at most

$$
\frac{3-2 \xi}{2 \xi-2} \frac{\xi+2}{3 \xi-1}=\frac{36-\sqrt{21}}{30}<2 .
$$

Notice that the expression on the left-hand side of $\left(42^{\prime}\right)$ is greater than 1 for each $t$ with $0 \leqslant t \leqslant 1$; hence using this procedure it is not possible to prove that $F_{3}(0) \div F_{3}(0)$ contains any interval. Finally, the expression on the left-hand side of $\left(43^{\prime}\right)$ is at most

$$
\frac{3-2 \xi}{2 \xi-2} \frac{\xi+2}{\xi+3}<2 \text {. }
$$

Proof of $\left(3^{\prime \prime}\right)$ from Theorem $2^{\prime \prime}$. We shall show that

$$
F_{2}(0)=L\left(\left[\frac{\sqrt{3}-1}{2}, \sqrt{3}-1\right]\right)
$$

and that $\mathrm{F}_{2}(0)$ satisfies the 4-condition.

We put

$$
I_{0}=\left[\frac{1}{2}(\sqrt{3}-1), \sqrt{3}-1\right]=[(0 ; \overline{2}, \overline{1}),(0 ; \overline{1}, \overline{2})] .
$$

We show that each of the intervals $I_{n}^{\left(i_{1} \ldots i_{n}\right)}$ will have endpoints of the type

$$
\left(0 ; a_{1}, \ldots, a_{r}, \overline{1,2}\right), \quad\left(0 ; a_{1}, \ldots, a_{r}, \overline{2,1}\right) .
$$

We remark, first of all, that $I_{0}$ has endpoints of type (45) (with $r=0$ ). If we have an interval with endpoints of type (45), then after deleting of the open interval with endpoints

$$
\left(0 ; a_{1}, \ldots, a_{r}, 1, \overline{1,2}\right),\left(0 ; a_{1}, \ldots, a_{r}, 2, \overline{2,1}\right)
$$

we get two closed intervals, one with endpoints $\left(0 ; a_{1} \ldots, a_{r}, 1, \overline{2,1}\right)$, $\left(0 ; a_{1}, \ldots, a_{r}, 1, \overline{1,2}\right)$, the other with endpoints $\left(0 ; a_{1}, \ldots, a_{r}, 2, \overline{2}, \overline{1}\right)$, $\left(0 ; a_{1}, \ldots, a_{r}, 2, \overline{1}, 2\right)$, thus both of type (45).

Analogously, it suffices to show that the following inequalities hold:

$$
\begin{aligned}
& \left|\frac{\left(0 ; a_{1}, \ldots, a_{r}, 1, \overline{1,2}\right)-\left(0 ; a_{1}, \ldots, a_{r}, 2, \overline{2,1}\right)}{\left(0 ; a_{1}, \ldots, a_{r}, 1, \overline{2}, 1\right)-\left(0 ; a_{1}, \ldots, a_{r}, 1, \overline{1,2}\right)}\right| \leqslant 3, \\
& \left|\frac{\left(0 ; a_{1}, \ldots, a_{r}, 1, \overline{1,2}\right)-\left(0 ; a_{1}, \ldots, a_{r}, 2, \overline{2,1}\right)}{\left(0 ; a_{1}, \ldots, a_{r}, 2, \overline{2,1}\right)-\left(0 ; a_{1}, \ldots, a_{r}, 2, \overline{1,2}\right)}\right| \leqslant 3 .
\end{aligned}
$$


We shall use the symbols $x_{r}, y_{r}, x_{r-1}, y_{r-1}, t$ in the same moaning as in the preceding proof. Instead of $\xi$ we introduce

$$
\eta=(\overline{1} ; 2)=\frac{1+\sqrt{3}}{2}
$$

Then we can write

$$
\begin{aligned}
& \left(0 ; a_{1}, \ldots, a_{r}, 1, \overline{1,2)}=\frac{(2 \eta-1) x_{r}+x_{r-1}}{(2 \eta-1) y_{r}+y_{r-1}},\right. \\
& \left(0 ; a_{1}, \ldots, a_{r}, 2, \overline{2,1)}=\frac{(\eta+1) x_{r}+x_{r^{\prime-1}}}{(\eta+1) y_{r}+y_{r-1}},\right. \\
& \left(0 ; a_{1}, \ldots, a_{r}, 1, \overline{2,1)}=\frac{\eta x_{r}+x_{r-1}}{\eta y_{r}+y_{r-1}},\right. \\
& \left(0 ; a_{1}, \ldots, a_{r}, 2, \overline{1,2}\right)=\frac{2 \eta x_{r}+x_{r-1}}{2 \eta y_{r}+y_{r-1}}
\end{aligned}
$$

After using (48) in (46) and (47), we can convince ourselves that the inequalities $(46),(47)$ are equivalent to

$$
\begin{aligned}
& \frac{2-\eta}{\eta-1} \frac{\eta+t}{\eta+1+t} \leqslant 3, \\
& \frac{2-\eta}{\eta-1} \frac{2 \eta+t}{2 \eta-1+t} \leqslant 3 .
\end{aligned}
$$

The expression on the left-hand side of $\left(46^{\prime}\right)$ attains its maximum in the interval $0 \leqslant t \leqslant 1$ at $t=1$ and the maximum is obviously less than 3 . The expression on the left-hand side of $\left(47^{\prime}\right)$ attrins its maximum at $t=0$ :

$$
\frac{2-\eta}{\eta-1} \frac{2 \eta}{2 \eta-1}=\sqrt{3}+1<3
$$

Notice that the expression on the leit-hand side of $\left(47^{\prime}\right)$ is greater than 2 if $0 \leqslant t \leqslant 1$ : hence it is not possible to show by this procedure that $F_{2}(0)+F_{2}(0)+F_{2}(0)$ contains any interval.

Analogous results concerning the products of continued fractions will be published later.

Note added in proof. The results of this paper were obtained in tho seminar of Prof. Jarnik in 1968. In connection with similar questions, T. W. Cusiok and. R. A. Lee have arrived recently at general statements about sums of discontinui which make it possible to give another proof of our results (see [2] and [3]).

\section{Bibliography}

[1] M. Hall, Jr., On the sum and product of continued fractions, Annals of Math. 48 (1947), pp. 966-993.

[2] T. W. Cusick and R. A. Lee, Sums of sets of continued fractions, Proc. AMS 30 (1971), pp. 241-246.

[3] T. W. Cusick, On M. Hall's continued fraction theorem, Proc. AMS (to appear, March 1973).

\section{DEPARTMENT OF MATHEMATICS}

OHIO STAATE UNIVERSTTY

Columbus, Ohio 